\title{
Cirugía reconstructiva compleja de la esclerosis cérvico-uretral tras prostatectomía radical, por fracaso de la solución endoscópica
}

\author{
Gaya Sopena JM, Palou Redorta J, Arañó Beltrán P, Caparrós Sariol J, Villavicencio Mavrich H.
}

Servicio de Urología. Fundació Puigvert, Barcelona.

Actas Urol Esp. 2008;32(7):752-755

\section{RESUMEN}

\section{CIRUGÍA RECONSTRUCTIVA COMPLEJA DE LA ESCLEROSIS CÉRVICO-URETRAL TRAS} PROSTATECTOMÍA RADICAL, POR FRACASO DE LA SOLUCIÓN ENDOSCÓPICA

La esclerosis de la unión cérvico-uretral es una de las complicaciones que pueden aparecer tras una prostatectomía radical, el tratamiento endoscópico suele ser resolutivo en la mayoría de los casos. Presentamos la reparación vía abierta de una esclerosis cérvico-uretral tras prostatectomía radical que no respondió al manejo endoscópico. La técnica quirúrgica constó de un primer tiempo perineal y un segundo tiempo con abordaje hipogástrico. A los 6 meses de esta compleja cirugía se colocó un esfinter artificial al paciente. Tras 3 años de seguimiento el paciente se encuentra asintomático y continente.

Palabras clave: Esclerosis Cérvico-uretral. Prostatectomía radical. Cirugía reconstructiva.

\section{ABSTRACT \\ COMPLEX RECONSTRUCTIVE SURGERY OF CERVICO-URETHRAL SCLEROSIS AFTER RADICAL PROSTATECTOMY, DUE ENDOSCOPIC SOLUTION FAILURE}

The sclerosis of the cervico-urethral union is one of the complications that may arise after a radical prostatectomy, in most cases, the endoscopic treatment usually solves it. We introduce repair by open approach of a sclerosis cervico-urethral after radical prostatectomy that did not respond to endoscopic management. The surgical technique had two times, the first perineal and a second time with hypogastric approach. After six months of this complex surgery an artificial sphincter was placed to patient. The patient is asymptomatic and continent after three years of follow up surgery.

Keywords: Cervicoe-urethral stenosis. Radical prostatectomy. Reconstructive surgery.

$\mathrm{L}$ a prostatectomía radical es una técnica habitual en el tratamiento del cáncer de próstata localizado.

Una de las complicaciones de esta técnica quirúrgica es la esclerosis de la unión cérvico-uretral, que oscila entre $3-12 \%{ }^{1}$. El manejo inicial de esta complicación suele ser conservador, con dilatación o tratamiento endoscópico (cervicotomía o uretrotomía interna), que resuelve un 70-90\% de los casos ${ }^{2}$.

Cuando fracasa el tratamiento conservador endoscópico la situación es compleja y de difícil manejo.

Se presenta un caso clínico complejo de estenosis uretrocervical tras prostatectomía radical que se resolvió de forma satisfactoria mediante cirugía abierta reconstructiva.

\section{CASO CLÍNICO}

Paciente varón de 65 años sin hábitos tóxicos ni antecedentes patológicos de interés se diagnosticó a raíz de PSA elevado de adenocarcinoma de próstata Gleason $(2+2)$ en Noviembre del 2001. Se le realizó prostatectomía radical en otro centro en Enero del 2002. Durante la cirugía surgieron problemas de control de hemostasia, con dos reintervenciones por sangrado en el postoperatorio inmediato y la necesidad de transfundir 39 concentrados de hematíes e ingreso en la unidad de cuidados intensivos durante 8 días. A los dos meses del alta, tras retirada de sonda uretral el paciente presentó retención aguda de orina que precisó de colocación de sonda de cistostomía. Después de dos intentos de cervicotomía 
endoscópica con resultados de una nueva retención de orina e infecciones urinarias de repetición se remitió a nuestro centro para valoración.

Se le practicó una uretrografía miccional/retrógrada que mostró estenosis a nivel de la unión cérvico-uretral (Fig. 1).

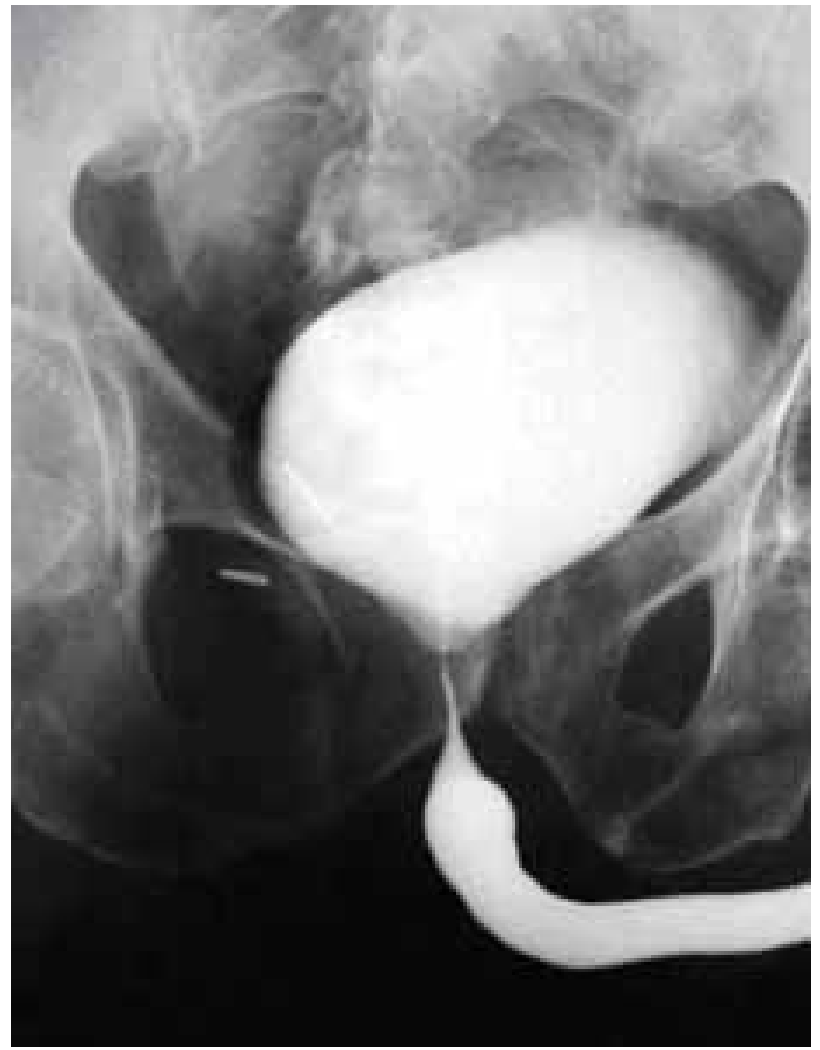

FIGURA 1. La uretrografia muestra una estenosis cérvico uretral con paso filiforme de contraste a uretra distal.

Se practicó un nuevo intento endoscópico con cervicotomía y resección del tejido fibroso de la zona de la anastomosis. La anatomía patológica informó de tejido fibroso con ausencia de tumor y los valores de PSA en sangre permanecían indetectables. A la semana de la retirada la sonda vesical presentó una nueva retención aguda de orina, por lo que se colocó de nuevo una sonda de cistostomía.

Por fracaso de las maniobras endoscópicas, a los diez meses se practicó un primer tiempo de cirugía reconstructiva. Primero, por vía perineal, en posición de litotomía se disecó uretra bulbar y membranosa hasta la zona fibrosa a nivel central del periné, con separación parcial de los cuerpos cavernosos, dejando una ligadura en el extremo y cierre de la herida perineal.
Después en un segundo tiempo por vía hipogástrica, en posición de decúbito supino se disecó la cara anterior de la vejiga, con resección parcial superior del arco pubiano, hasta llegar al cuello vesical y zona fibrótica cérvico-uretral. Se extirpó la zona fibrótica y se realizó una sutura uretrovesical con puntos simples. Se dejó una sonda de cistostomía y otra uretral.

A las tres semanas se retiró la sonda uretral tras realización de cistografía que confirmó la estanquedad de la sutura y el buen paso por la zona anastomótica (Fig. 2).

A los seis meses se colocó un esfínter artificial (AMS 800) a nivel de uretra bulbar con activación a los dos meses (Fig. 3).

El paciente actualmente se encuentra continente y no presenta residuo postmiccional.

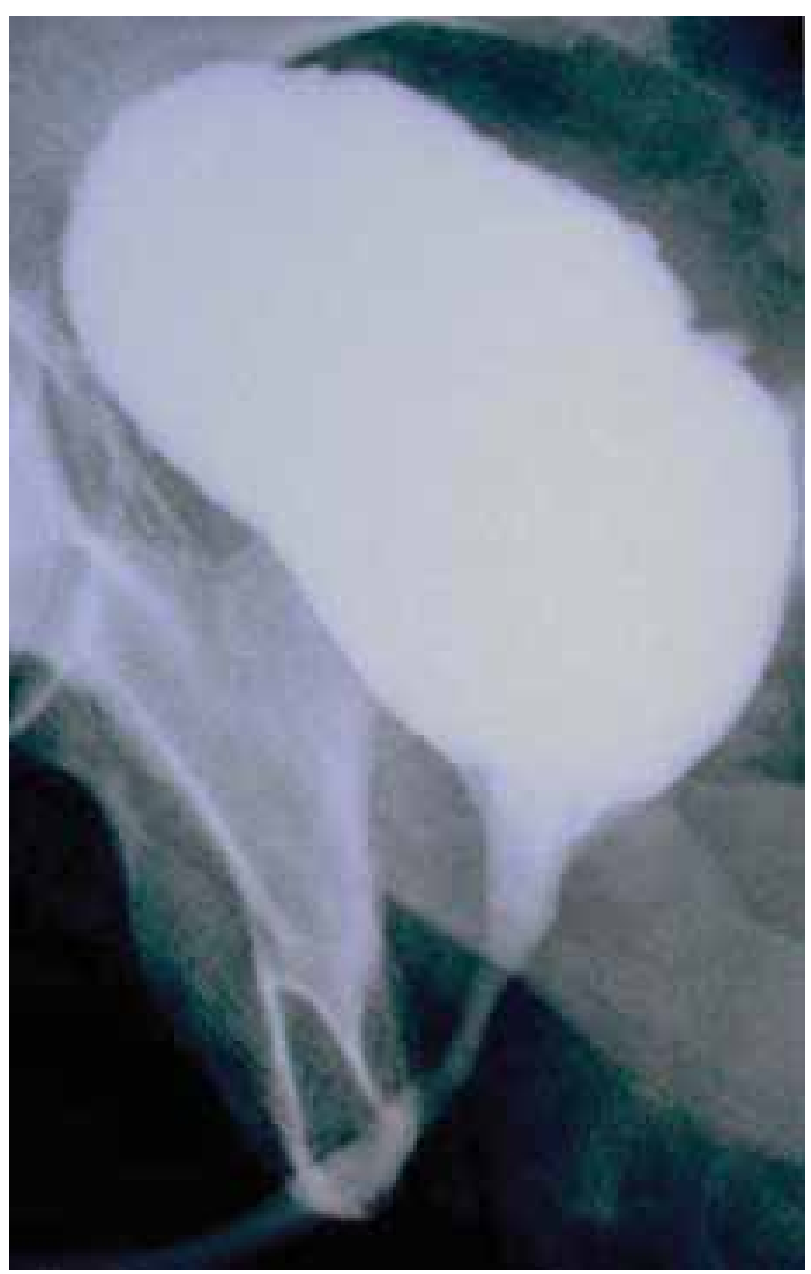

FIGURA 2. Cistografia de relleno/uretrografia miccional donde no se observan extravasados y buen paso de contraste sin observarse segmentos estenóticos. 


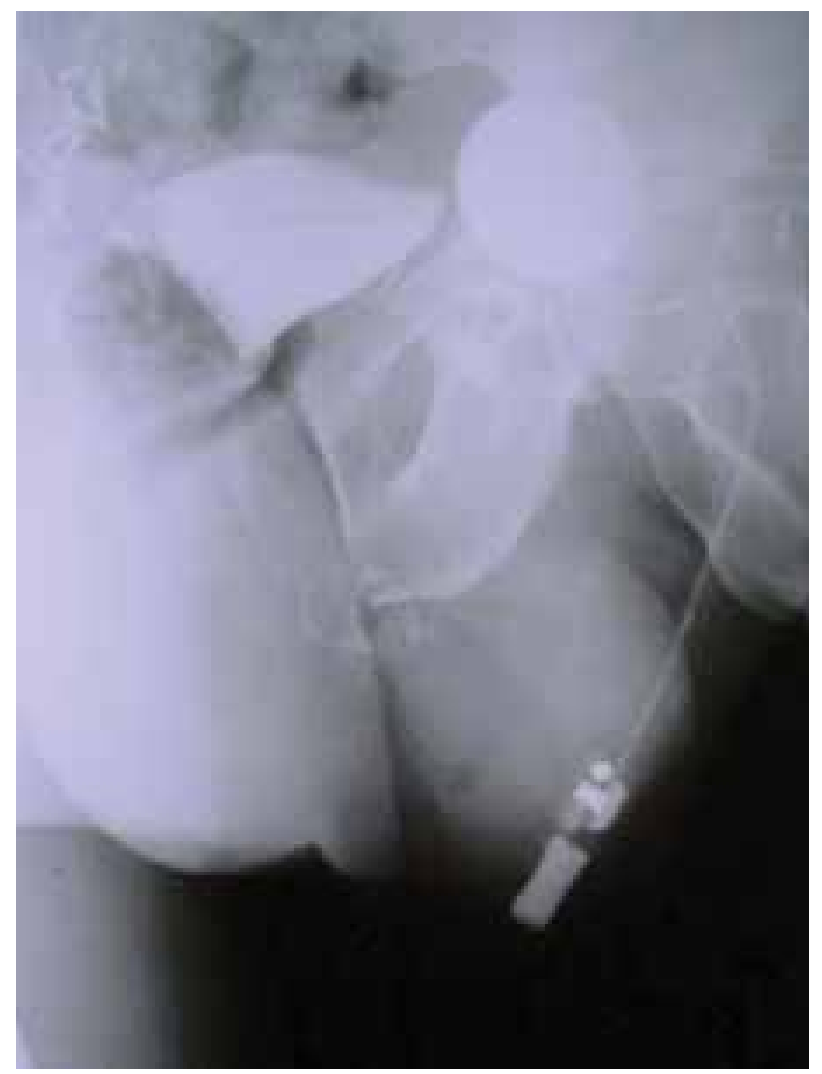

FIGURA 3. Rx adomino-perineal que muestra esfinter normocolocado a nivel de uretra bulbar con presencia de resevorio hipogástrico.

\section{DISCUSION}

La esclerosis de la unión cérvico-uretral es una de las complicaciones que pueden aparecer tras la cirugía radical de próstata.

Los factores de riesgo que parece se asocian con la aparición de este tipo de estenosis son: el extravasado de orina en la zona de la anastomosis, una resección transuretral de próstata previa, la reconstrucción del cuello vesical de forma esclerótica, la movilización excesiva de la uretra bulbar ${ }^{3}$, y el antecedente de tratamiento radioterápico previo ${ }^{4}$ (cuando la prostatectomía radical se realiza a modo de salvaje, tras fracaso de la radioterapia). En relación al sangrado existe controversia si el riesgo de esclerosis es mayor cuando este es intraoperatorio o cuando sucede en el postoperatorio ${ }^{5}$. Los factores que no parecen influir son: el extravasado en la cistografía inicial y el tiempo de catéter uretral postoperatorio $^{6}$.

El tratamiento conservador con dilataciones o tratamiento endoscópico suele ser suficiente para solucionar el 70-90 \% de los casos. Se debe usar en estos casos siempre el cuchillete frío, el uso de electrocoagulación aumenta el riesgo de incontinencia urinaria ${ }^{7}$.

Cuando el tratamiento endoscópico fracasa, como sucedió en el caso descrito, se deben realizar abordajes quirúrgicos más complejos y de los que se dispone poca experiencia por el excelente resultado del manejo endoscópico. Estos tratamientos más agresivos exponen al paciente a una mayor morbilidad que los tratamientos conservadores ${ }^{8}$.

La pubectomía en el procedimiento combinado es útil ya que facilita el procedimiento quirúrgico y, además, disminuye el tiempo total de la cirugía.

La colocación del esfínter artificial es una solución al problema de la lesión del esfínter uretral en estos casos complejos, así como en la incontinencia urinaria severa post prostatectomía radical ${ }^{9}$.

Otra opción para el tratamiento de estas estenosis es la colocación de un stent uretral permanente. Parece que este es un tratamiento efectivo, en casos muy bien seleccionados, pero no exento complicaciones $^{10-12}$.

\section{CONCLUSIONES}

El tratamiento de elección de esta complicación es endoscópico. Sólo cuando éste fracasa, se puede realizar una cirugía abierta reconstructiva. La cirugía abierta es una técnica compleja, que no está exenta de morbilidad y que requiere experiencia en cirugía pélvica y perineal.

La colocación de un esfínter artificial es una opción segura y exitosa al problema de la incontinencia urinaria después de la resección del segmento estenótico.

\section{REFERENCIAS}

1. Dalkin BL. Endoscopic evaluation and treatment of anastomotic strictures after radical retropubic prostatectomy. J Urol.1996; 155(1):206-209.

2. Gómez Veiga F, González Martín M, Sánchez Rodríguez J, Chantada Abal V, Busto Castañón L, Sousa Escandón A. Endoscopic cervicotomy in post-prostatectomy sclerosis of the bladder neck.Arch Esp Urol. 1991 Jul-Aug;44(6):727-732;

3. Borboroglu PG, Sands JP, Robert JL, Amling CL. Risk factors for vesicourethral anastomotic stricture after radical prostatectomy. Urology. 2000;56(1):96-100.

4. Pisters LL, English SF, Scott SM, Westney OL, Dinney CP, Mc Guire EJ. Salvaje prostatectomy with continent catheterizable urinary reconstruction: a novel approach to recurrent prostate cancer after radiation therapy. J Urol. 2000;163(6):1771-1774.

5. Surya BV, Provet J, Johanson KE, Brown J. Anastomotic strictures following radical prostatectomy: risk factors and management. J Urol. 1990;143(4):755-758. 
6. Huang G, Lepor H. Factors predisposing to the development of anastomotic strictures in a single-surgeon series of radical retropubic prostatectomies. BJU Int. 2006;97(2):255-258.

7. Rosales A, Vicente J. Cirugía endoscópica del cuello y la uretra. Tratado de endourología. Ed.Pulso: 6: 413, 1996.

8. Schlossberg S, Jordan G, Schellhammer P. Repair of obliterative vesicourethral stricture after radical prostatectomy: a technique for preservation of continence. Urology. 1995;45(3):510513

9. Wessells H, Morey AF, McAninch JW. Obliterative vesicourethral strictures following radical prostatectomy for prostate cancer: reconstructive armamentarium. J Urol. 1998;160(4):13731375.

10. Zivan I, Stein A. New modality for treatment of resistant anastomotic strictures after radical prostatectomy: Urolume urethral stent. J Endourol. 2001;15(8):869-871.
11. Chiou RK, Howe S, Morton JJ, Grune MT, Taylor RJ. Treatment of recurrent vesicourethral anastomotic stricture after radical prostatectomy with endouretroplasty. Urology. 1996; 47(3):422-425.

12. Ricciotti G, Bozzo W, Perachino M, Pezzica C, Puppo P. Heatexpansible permanent intraurethral stents for benign prostatic hyperplasia and urethral strictures. J Endourol. 1995;9(5): 417-422.

Correspondencia autor: Dr. J.M ${ }^{\mathrm{a}}$ Gaya Sopena

Servicio de Urología. Fundació Puigvert.

Cartagena 340-350, 08025 Barcelona. Tel.: 934169700

E-mail autor: 36178jgs@comb.es

Información artículo: Nota Clínica

Trabajo recibido: enero 2007

Trabajo aceptado: febrero 2007 